\title{
Optoelectronic properties of single-layer, double- layer, and bulk tin sulfide: A theoretical study
}

\section{Citation}

Tritsaris, Georgios A., Brad D. Malone, and Efthimios Kaxiras. 2013. "Optoelectronic Properties of Single-Layer, Double-Layer, and Bulk Tin Sulfide: A Theoretical Study." Journal of Applied Physics 113 (23) (June 21): 233507. doi:10.1063/1.4811455.

\section{Published Version}

10.1063/1.4811455

\section{Permanent link}

http://nrs.harvard.edu/urn-3:HUL.InstRepos:33371487

\section{Terms of Use}

This article was downloaded from Harvard University's DASH repository, and is made available under the terms and conditions applicable to Other Posted Material, as set forth at http:// nrs.harvard.edu/urn-3:HUL.InstRepos:dash.current.terms-of-use\#LAA

\section{Share Your Story}

The Harvard community has made this article openly available.

Please share how this access benefits you. Submit a story.

Accessibility 


\title{
Optoelectronic properties of single-layer, double-layer, and bulk tin sulfide: A theoretical study
}

\author{
Georgios A. Tritsaris, ${ }^{1}$ Brad D. Malone,${ }^{1}$ and Efthimios Kaxiras ${ }^{1,2, a)}$ \\ ${ }^{1}$ School of Engineering and Applied Sciences, Harvard University, Cambridge, Massachusetts 02138, USA \\ ${ }^{2}$ Department of Physics, Harvard University, Cambridge, Massachusetts 02138, USA
}

(Received 2 May 2013; accepted 3 June 2013; published online 18 June 2013)

\begin{abstract}
$\mathrm{SnS}$ is a metal monochalcogenide suitable for use as absorber material in thin film photovoltaic cells. Its structure is an orthorhombic crystal of weakly coupled layers, each layer consisting of strongly bonded Sn-S units. We use first-principles calculations to study model single-layer, double-layer, and bulk structures of $\mathrm{SnS}$ in order to elucidate its electronic structure. We find that the optoelectronic properties of the material can vary significantly with respect to the number of layers and the separation between them: the calculated band gap is wider for fewer layers $(2.72 \mathrm{eV}$, $1.57 \mathrm{eV}$, and $1.07 \mathrm{eV}$ for single-layer, double-layer, and bulk SnS, respectively) and increases with tensile strain along the layer stacking direction (by $\sim 55 \mathrm{meV} / 1 \%$ strain). (C) 2013 AIP Publishing LLC. [http://dx.doi.org/10.1063/1.4811455]
\end{abstract}

\section{INTRODUCTION}

Thin film photovoltaic (PV) cells is a technology of increasing importance for direct conversion of solar energy into electricity. ${ }^{1,2}$ The conversion efficiency of the PV depends critically on the optoelectronic properties of the absorber layer, ${ }^{3}$ and PV cells based on the chalcogen-

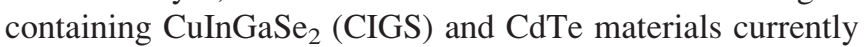
have the highest efficiencies for this type of materials exceeding 15\%. ${ }^{4,5} \mathrm{SnS}$ (Refs. 6-10) is also being considered as absorber material for thin film PV cells: ${ }^{1-15}$ it is a semiconductor with a layered structure, an optical band gap of $\sim 1.3 \mathrm{eV}$, which is in the optimal range for solar cells (1.1-1.5 eV), and high absorption in the visible (absorption coefficient $>10^{4} \mathrm{~cm}^{-1}$ ). Moreover, $\mathrm{SnS}$ is based on nontoxic elements in contrast to CIGS and CdTe. Although theory indicates that the efficiency of solar cells based on $\mathrm{SnS}$ could match that of CIGS and CdTe-based cells, in practice much lower efficiencies have been achieved $(<2 \%)$, leaving room for improvement in the absorber and the design of the PV device. ${ }^{13,16-20}$ General strategies for tuning the properties of the material, also relevant to the fabrication of novel electronic devices, include the use of atomically thin compounds $^{21-24}$ and the application of mechanical strain. ${ }^{25-27} \mathrm{SnS}$ has been extensively investigated in its bulk form ${ }^{28-34}$ but few-layer and single-layer structures remain largely unexplored. ${ }^{8,35}$

The measured properties of SnS samples depend on the synthesis route. ${ }^{9,36-39}$ Theory and atomistic simulation can contribute significantly in clarifying how the properties of $\mathrm{SnS}$ and other layered semiconductors depend on the atomicscale structural features. ${ }^{12,25,27,30,32,40,41}$ Here, we use firstprinciples calculations based on density functional theory and the GW approximation to the electron self-energy to study trends in the electronic and optical properties of model single-layer, double-layer, and bulk structures of $\mathrm{SnS}$. Specifically, we calculate the indirect and direct band gaps

a)e-mail: kaxiras@physics.harvard.edu and the dielectric functions, which depend on the number of layers and the separation between them: the direct band gap narrows in going from single-layer to bulk SnS or by applying tensile strain along the layer stacking direction. Tensile strain also results in a dramatic increase in the electron effective mass for the double-layer and bulk SnS. These findings point to possible approaches for tuning the electronic structure of $\mathrm{SnS}$ for improved performance in optoelectronic applications.

\section{MODELS AND METHODS}

We used an orthorhombic unit cell with $4 \mathrm{Sn}$ and $4 \mathrm{~S}$ atoms to model the bulk structure of SnS (Fig. 1), which is in the space group Pnma. The layers are arranged perpendicular to the $a$-axis and are held together by weak forces in contrast to strong intralayer bonds. ${ }^{42}$ For the model double-layer structure of $\mathrm{SnS}$, the unit cell of bulk $\mathrm{SnS}$ was augmented by a region of vacuum with width $8 \AA$ in the direction perpendicular to the layers, and for the model single-layer structure of $\mathrm{SnS}$ the unit cell consists of one layer of $\mathrm{SnS}$ with $5 \AA$ of vacuum on each side. The choice of vacuum regions between the slabs was guided by the requirement that the wave functions vanish smoothly at the edge of the unit cell, as would be required in a model of the isolated system in each case.

For total energy calculations we used the GPAW code, ${ }^{43}$ a grid-based approach using the projected augmented-wave
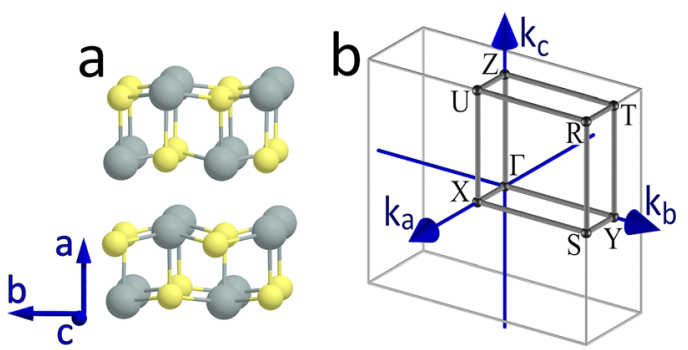

FIG. 1. Structural model of orthorhombic tin sulfide ( $\mathrm{SnS})$. Gray and yellow balls represent $\mathrm{Sn}$ and $\mathrm{S}$, respectively. (a) Unit cell, repeated twice in the $b c$-plane, and (b) corresponding Brillouin zone. 
method (an all-electron method using the frozen core approximation). ${ }^{44}$ For structural optimization, we elected to use the local density approximation (LDA) for the description of exchange and correlation (GPAW setup release v0.8). This approach has been shown to describe SnS with satisfactory (albeit fortuitous) accuracy compared to experiment ${ }^{12}$ and with much less computational effort compared to approaches that aim at higher accuracy, for instance with the use of hybrid functionals. ${ }^{12,30,32,45}$ Thus, it provides a reasonable way to address the elastic properties of the material in the various forms we considered here. On the other hand, LDA typically underestimates the fundamental band gap of semiconductors and insulators. To obtain reliable band structures, we calculated the relaxed structures using the GLLB-SC functional, an orbital-dependent functional based on the Gritsenko-Leeuwen-Lenthe-Baerends potential ${ }^{46,47}$ modified with elements from the revised Perdew-BurkeErnzerhof functional, PBEsol (SC: solid and correlation). ${ }^{48}$ This functional accounts explicitly for the discontinuity in the derivative of (semi-)local exchange-correlation functionals such as LDA, and it has been successfully used to predict the band gap of prototypical bulk and low-dimensional semiconductors and insulators. ${ }^{47,49,50}$ The discontinuity depends on the space coordinate but a constant value is obtained by first-order perturbation theory. More details on the formalism are given in the work of Kuisma et al ${ }^{47}$ For $k$-space integration, a Monkhorst-Pack mesh of $1 \times 8 \times 8 k$-points was used for the single-layer and double-layer structures and a mesh of $4 \times 8 \times 8 k$-points was used for the bulk structure. The correction to the Kohn-Sham single-particle energies is applied as a rigid shift in the valence band energies by the calculated discontinuity. For example, for crystalline diamond this method predicts an indirect band gap of $5.46 \mathrm{eV}$ (5.5 $\mathrm{eV}$ in experiment) from a Kohn-Sham band gap of $4.17 \mathrm{eV}$ and discontinuity $\Delta_{\mathrm{xc}}=1.29 \mathrm{eV}$. To validate this simple approach for the case of $\mathrm{SnS}$, we have performed calculations for the electronic structure based on the GW approximation to the electron self-energy, which gives reliable results across a very wide range of materials. ${ }^{51,52}$ These calculations were performed using the BerkeleyGW package $^{53}$ with wavefunctions and energies obtained from the Quantum-ESPRESSO code. ${ }^{54}$ The calculation of the selfenergy was performed on a uniform grid of size $2 \times 6 \times 5$ in the Brillouin zone. The unshifted grid of $2 \times 6 \times 5$ was used both in the summation over $q$-vectors as well as the $k$-grid on which the quasiparticle energies were computed. A quasiparticle calculation was also performed on a shifted grid of $k$-vectors so as to represent accurately the valence band maximum (VBM), which is not contained in the unshifted grid. Particular care was taken to achieve adequate convergence for the slowly-converging summations over empty states. Our calculations use 2600 empty states in the evaluation of the Coulomb-hole contribution to the self-energy, 1500 empty states in the evaluation of the polarizability, and a static dielectric matrix with cutoff-energy of 26 Rydberg was extended to finite-frequencies using a plasmon-pole model. ${ }^{51}$

Dielectric functions were calculated within the framework of time-dependent density functional theory ${ }^{55}$ using the bootstrap approach of Sharma, Gross and co-workers, ${ }^{56}$ which is based on an approximate expression for the exchange-correlation kernel and its self-consistent numerical derivation. This approach has been demonstrated to give optical absorption spectra in good agreement with experiment, capturing excitonic features with small computational effort compared to methods based on the GW/Bethe-Salpeter formalism. ${ }^{56,57}$ For these calculations, we used the corrected Kohn-Sham single-particle energies that were obtained with the GLLB-SC approach mentioned earlier. Empty electronic bands up to $50 \mathrm{eV}$ above the Fermi level were included and the dielectric function was calculated using a planewave basis with an energy cut-off of $150 \mathrm{eV}$. The position and overall shape of the main features of the dielectric function such as shoulders and peaks were converged with respect to the mentioned parameters as well as the $k$-point mesh.

\section{RESULTS AND DISCUSSION}

For the bulk structure of SnS, after optimization of the unit cell with fixed scaled atomic positions, the lattice constants are $\mathrm{a}_{0}=11.12 \AA, \mathrm{b}_{0}=3.95 \AA$, and $\mathrm{c}_{0}=4.24 \AA$, in reasonable agreement with experiment (the measured lattice constants at $295 \mathrm{~K}$ are $\mathrm{a}_{0}=11.20 \AA, \mathrm{b}_{0}=3.99 \AA$, and $\left.\mathrm{c}_{0}=4.33 \AA\right) .{ }^{58}$ Optimization of the atomic positions reduces the total energy per atom by only $1 \mathrm{meV}$. The distance between the closest two $\mathrm{Sn}$ atoms in two neighboring layers in the optimized cell, projected on the $a$-axis, is $2.62 \AA$. The unit cell of the model single-layer structure of $\mathrm{SnS}$ consists of one layer. To confirm that the single-layer structure is stable in its own right when separated from the rest of the system, we calculated the phonon frequencies at the $\Gamma$-point using the frozen phonon approximation and we found no imaginary values. As a check of stability at room temperature, we performed a molecular dynamics simulation for 1.0 ps in the microcanonical ensemble $(\mathrm{N}, \mathrm{V}$, and $\mathrm{E})$ using the relatively high temperature of $600 \mathrm{~K}$ and a time step of 1.5 fs, and obtained the average Sn-Sn, S-S, and S-Sn bond lengths in a single unit cell from configurations in the last $0.5 \mathrm{ps}$ with a sampling of $1 / 50 \mathrm{fs}^{-1}$. Compared to the ground state, the deviations for Sn-Sn, S-S and S-Sn bond lengths at $600 \mathrm{~K}$ are $2 \%, 2 \%$, and $6 \%$, respectively.

Fig. 2(a) shows the band structure of our model singlelayer SnS, calculated along the high-symmetry directions $\mathrm{Y}-\mathrm{\Gamma}-\mathrm{Z}-\mathrm{T}-\mathrm{Y}$ in the Brillouin zone. There are 40 electrons in the unit cell, which are accommodated by 20 bands but only bands that lie within a few $\mathrm{eV}$ from the band gap are shown here. The contributions of Sn- and S-orbitals are shown in separate panels in Fig. 2(a). In Figs. 2(a)-2(c), the energy eigenvalues are color-coded with respect to the relative contribution of the $s-, p-$, and $d$-levels of Sn, and $s$ - and $p$ - levels of $\mathrm{S}$, and the symbol size is proportional to the relative total contribution of the element. For each element, the contribution of its orbital is calculated from the overlap between the corresponding projector functions and the pseudo wavefunction at each $k$-point. ${ }^{43}$ For instance, both Sn and S contribute to the electronic states close to the valence band maximum, which is located along the $\Gamma-Z$ direction. We will refer to this $k$-point as $\bar{Z}_{\mathrm{v}}$, following the notation $\overline{\mathrm{K}}_{\mathrm{b}}$, where " $\overline{\mathrm{K}}$ " signifies the direction in Brillouin zone along which the $k$-point 

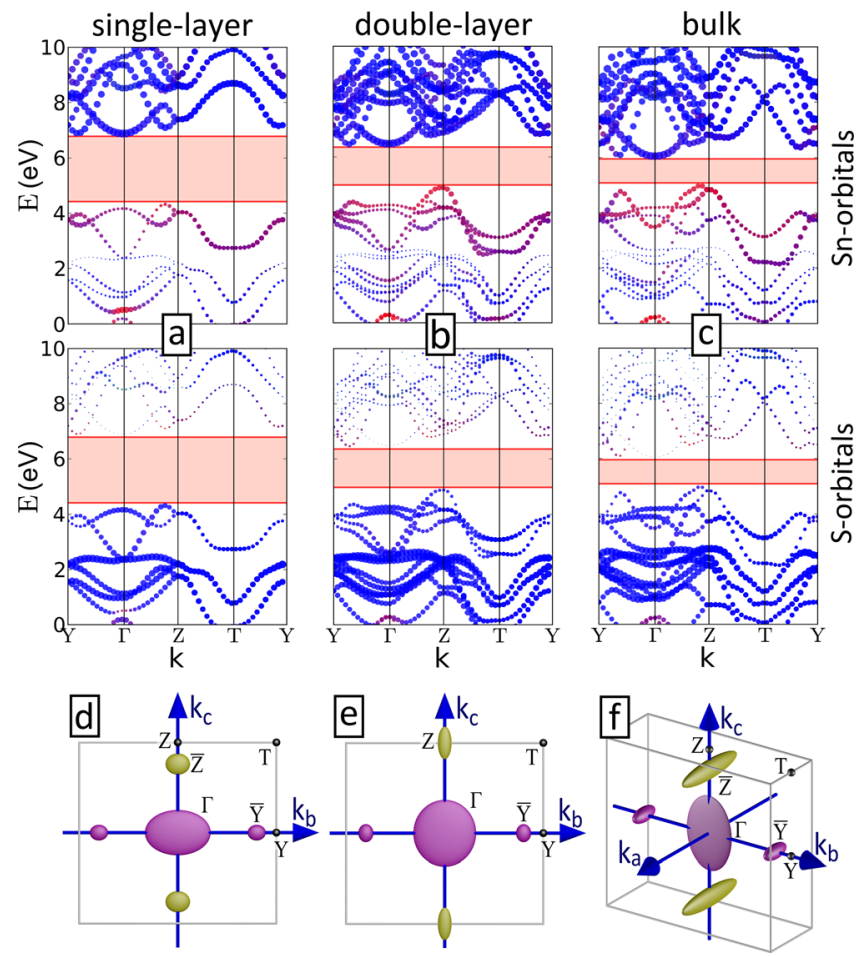

FIG. 2. Band structure for model (a) single-layer, (b) double-layer and (c) bulk SnS, and [(d)-(f)] corresponding Brillouin zone and constant energy surfaces close to the edge of the conduction (purple) and valence (yellow) band. In $[(a)-(c)]$ the band structure is shown along the path that traces the edge of the Brillouin zone in a clock-wise fashion. At each $k$-point the energy eigenvalues are color-coded with respect to the relative contribution of the $s$ - (red), $p$ - (blue), and $d$ - (green) levels of Sn (top panel), and $s$ - and $p$-levels of S (bottom panel), and the symbol size is proportional to the relative total contribution of the element. The shaded horizontal strip delimits the band gap. The lowest-energy level of each band structure is shifted to $-17 \mathrm{eV}$ (level is not shown).

is located, namely "X", "Y," or " $Z$ " for $\Gamma-X, \Gamma-Y$, or $\Gamma-Z$ direction, and the subscript " $b$ " denotes the valence (v) or conduction (c) band. There are two competing $k$-points for the conduction band minimum (CBM), both dominated by $p$ Sn-levels: one is $\Gamma$ and the other is located along the $\Gamma-Y$ direction, $\bar{Y}_{c}$, both within a small energy range of $3 \mathrm{meV}$. The calculated minimum Kohn-Sham band gap, the derivative discontinuity, and the (corrected) indirect band gap are $1.78 \mathrm{eV}, 0.79 \mathrm{eV}$, and $2.57 \mathrm{eV}$ (Table I). The minimum direct band gap is $2.72 \mathrm{eV}$, located at $\Gamma$ (along the $\Gamma-\mathrm{Y}$ and $\Gamma-\mathrm{Z}$ directions the direct band gaps are $2.93 \mathrm{eV}$ and $2.77 \mathrm{eV}$, respectively). These large band gaps suggest that singlelayer $\mathrm{SnS}$ is transparent for the largest wavelengths in the visible.

TABLE I. Kohn-Sham band gap $\left(\mathrm{E}_{\mathrm{KS}}\right)$, derivative discontinuity $\left(\Delta_{\mathrm{xc}}\right)$, indirect $\left(\mathrm{E}_{\mathrm{g}}^{(\mathrm{i})}\right)$, and direct $\left(\mathrm{E}_{\mathrm{g}}^{(\mathrm{d})}\right)$ band gaps, all in $\mathrm{eV}$, and the electron $\left(\overline{\mathrm{m}}_{\mathrm{e}}\right)$ and hole $\left(\overline{\mathrm{m}}_{\mathrm{h}}\right)$ effective masses in units of bare electron mass at $k$-points involved in indirect transitions along the $b$ - and $c$-direction (see also Fig. 2).

\begin{tabular}{lcccccccc}
\hline \hline & & & & & $\overline{\mathrm{m}}_{\mathrm{e}}^{(\mathrm{b})} \overline{\mathrm{m}}_{\mathrm{e}}^{(\mathrm{c})}$ & $\overline{\mathrm{m}}_{\mathrm{e}}^{(\mathrm{b})} \overline{\mathrm{m}}_{\mathrm{e}}^{(\mathrm{c})}$ & $\overline{\mathrm{m}}_{\mathrm{h}}^{(\mathrm{b})} \overline{\mathrm{m}}_{\mathrm{h}}^{(\mathrm{c})}$ \\
& $\mathrm{E}_{\mathrm{KS}}$ & $\Delta_{\mathrm{xc}}$ & $\mathrm{E}_{\mathrm{g}}^{(\mathrm{i})}$ & $\mathrm{E}_{\mathrm{g}}^{(\mathrm{d})}$ & at $\overline{\mathrm{Y}}$ & at $\bar{\Gamma}$ & at $\overline{\mathrm{Z}}$ \\
\hline Single-layer & 1.78 & 0.79 & 2.57 & 2.72 & 0.230 .20 & 1.110 .77 & 0.310 .27 \\
Double-layer & 1.11 & 0.47 & 1.57 & 1.98 & 0.190 .23 & 1.061 .13 & 0.230 .55 \\
Bulk & 0.75 & 0.32 & 1.07 & 1.32 & 0.160 .20 & 0.911 .36 & 0.190 .36 \\
\hline \hline
\end{tabular}

We consider next a model double-layer structure of $\mathrm{SnS}$. We calculate the binding energy of the two layers, or equivalently the energy needed to separate them, to be $56 \mathrm{meV}$ per unit cell. Fig. 2(b) shows the band structure of the doublelayer. In going from the single-layer to the double-layer $\mathrm{SnS}$ the electronic bands split: for instance, the band splitting obtained from the energy difference between the bands located at $\sim 3 \mathrm{eV}$ near $\mathrm{T}$ is $\sim 0.5 \mathrm{eV}$. The valence band maximum lies at $\mathrm{Z}$, and as in the case of the single-layer there are two competing $k$-points very close in energy for the conduction band minimum, namely $\Gamma$ and $\bar{Y}_{c}$, with $\bar{Y}_{c}$ lower in energy than $\Gamma$ by $20 \mathrm{meV}$. The calculated indirect band gap is $1.57 \mathrm{eV}\left(\Delta_{\mathrm{xc}}=0.47 \mathrm{eV}\right), 1.50 \mathrm{eV}$ lower than the band gap of the single-layer SnS. Finally, the minimum direct band gap, located at $\bar{Z}$, is $1.98 \mathrm{eV}$, that is, $0.74 \mathrm{eV}$ lower than that for the single-layer (along the $\Gamma$-Y direction the direct band gap is $2.11 \mathrm{eV}$ ).

To understand better the origin of the band gap but also to relate our study to previous experimental and theoretical work in the literature, we calculated the bulk structure of $\mathrm{SnS}$. The binding energy of the layers is $56 \mathrm{meV}$ per unit cell. The binding energy is equal to that for the double-layer showing that the interaction is strictly between nearest neighbor layers. The hybridization of bands in bulk SnS [Fig. 2 (c)] is much more pronounced than in the double-layer structure: compare for example the dispersion of bands in the $\Gamma$-Y directions between the two different structures. The valence band maximum for the bulk structure is identified as a sharp peak at $\bar{Z}_{\mathrm{v}}$ and consistent with the single-layer and double-layer $\mathrm{SnS}$ the two competing $k$-points for the conduction band minimum are $\Gamma$ and $\bar{Y}_{c}$. The difference in energy between these $k$-points is only $40 \mathrm{meV}$, with the value at $\Gamma$ lying lower in energy. Previous theoretical studies have reported $\overline{\mathrm{Y}}_{\mathrm{c}}$ as the conduction band minimum. ${ }^{12,30} \mathrm{~A}$ possible reason for this discrepancy is that spin-orbit interactions are not treated in our model, the inclusion of which has been suggested to redefine the location of the band gap. ${ }^{30}$ However, as we show later, the shapes of the conduction and valence bands are highly sensitive to the interlayer distance, therefore, we expect strain effects to dominate spin-orbit interaction.

We proceed to calculate the effective mass of electrons at $\Gamma$ and at $\bar{Y}_{c}$ [Figs. 2(d)-2(f) and Table I]. For the bulk structure, the effective mass at $\bar{Y}_{c}$ is $0.45 m_{0}$ (the LDA value is $\left.0.35 \mathrm{~m}_{0}\right), 0.16 \mathrm{~m}_{0}(0.16)$, and $0.20 \mathrm{~m}_{0}(0.18)$ along the $a$-, $b$-, and $c$-axis, where $\mathrm{m}_{0}$ is the bare electron mass. At $\Gamma$ the effective masses are $0.10 \mathrm{~m}_{0}(0.09), 0.91 \mathrm{~m}_{0}(0.94)$, and 1.36 $\mathrm{m}_{0}(1.45)$. Assuming that the conduction band minimum is at $\overline{\mathrm{Y}}_{\mathrm{c}}$, our calculations are in very good agreement with previous experimental and theoretical work. ${ }^{11,12,42,59}$ For instance, Vidal, Zunger and co-workers ${ }^{12}$ report $0.5 \mathrm{~m}_{0}, 0.13 \mathrm{~m}_{0}$, and $0.20 \mathrm{~m}_{0}$ for the effective mass of electrons using calculations based on the GW method. We also find that the electron effective mass along the $b$ - and $c$-axis at $\overline{\mathrm{Y}}_{\mathrm{c}}$ among all structural models varies little, being $0.23 \mathrm{~m}_{0}$ and $0.20 \mathrm{~m}_{0}$ for the single-layer, and $0.19 \mathrm{~m}_{0}$ and $0.23 \mathrm{~m}_{0}$ for the double-layer.

For the bulk structure of $\mathrm{SnS}$, the indirect band gap is $1.07 \mathrm{eV}\left(\Delta_{\mathrm{xc}}=0.32 \mathrm{eV}\right)$, which is lower by $1.50 \mathrm{eV}$ and $1.00 \mathrm{eV}$ than the band gap of the single-layer and 

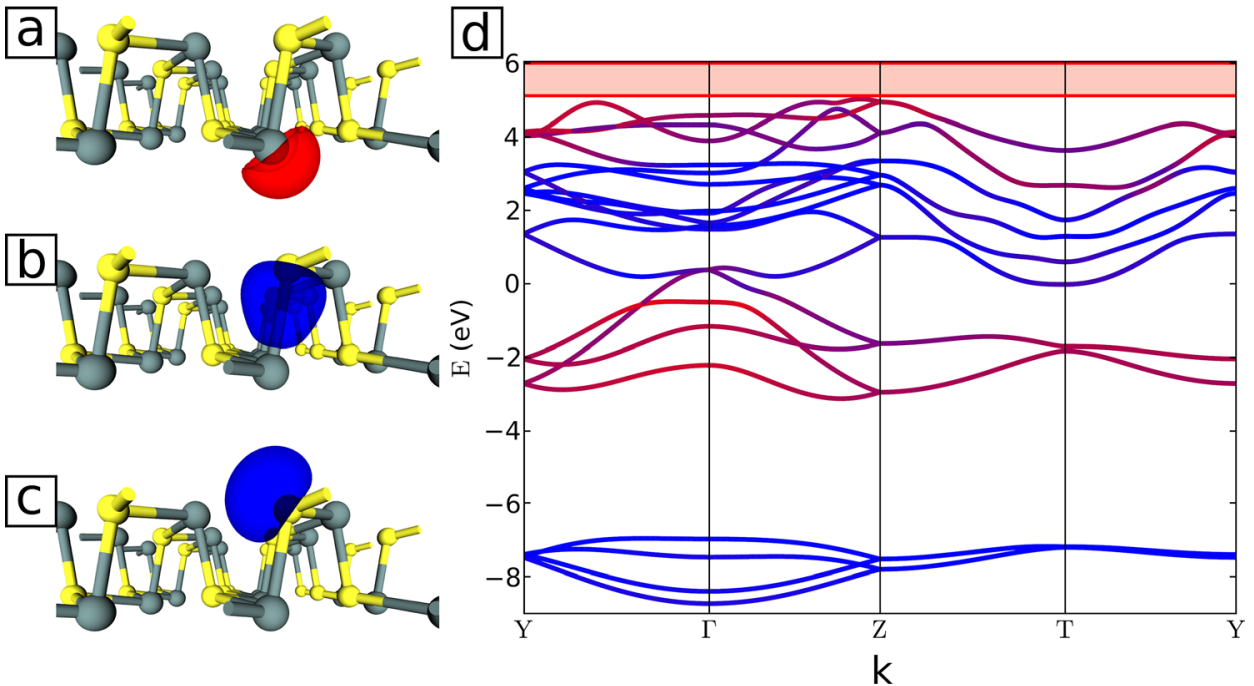

FIG. 3. Maximally-localized Wannier functions constructed from the occupied valence states in bulk $\mathrm{SnS}$, describing (a) Sn lone-pair states of which there are 4, one for each $\mathrm{Sn}$ atoms, (b) bonding states of which are 12, three for each Sn-S pair, and (c) states localized around the $\mathrm{S}$ atoms, of which there are 4. (d) Projection of the Wannier functions onto the Bloch band structure. At each $k$-point, the energy eigenvalues are color-coded with respect to the relative contribution of the Sn lone-pair states (red), and the bonding and S-states states (blue). double-layer structure, respectively. Corrections of $0.38 \mathrm{eV}$ (Ref. 29) and $0.32 \mathrm{eV}$ (Ref. 12) have been reported in studies based on the GW method. The band gap calculated with LDA is $0.58 \mathrm{eV}$, which amounts to almost half of the quasiparticle band gap calculated with GLLB-SC. In order to check the validity of the magnitude of the quasiparticle gap calculated with the GLLB-SC functional and the validity of a wave vector-independent rigid shift of eigenvalues, we also performed a calculation of the quasiparticle gap within the GW approximation to the self-energy. The GW calculations find that the LDA band gap of $0.59 \mathrm{eV}$, calculated with the plane-wave basis, is increased to $1.17 \mathrm{eV}$ upon the addition of the quasiparticle corrections. This quasiparticle gap is similar to that calculated using the GLLB-SC functional. Furthermore, an analysis of the size of the valence and conduction band GW corrections over the unshifted $2 \times 6 \times 5$ grid reveals that the individual quasiparticle shifts for wavevectors in the valence and conduction band are tightly clustered within $\pm 50 \mathrm{meV}$ of the average shift of the valence and conduction band, respectively. This fact gives us confidence that the rigid gap correction applied using the GLLB-SC functional provides a reasonable representation of the quasiparticle spectrum. Finally, the minimum direct band gap is calculated to be $1.32 \mathrm{eV}$ at $\overline{\mathrm{Y}}_{\mathrm{c}}$ (along the $\Gamma-\mathrm{Z}$ direction the direct band gap is $1.62 \mathrm{eV})$. Overall, the calculated band gaps are in very good agreement with previously published values. $^{12,17,29,30,34}$

In order to better understand the bonding chemistry within a layer of $\mathrm{SnS}$, we analyze the maximally-localized Wannier functions of the valence band manifold, which has proven useful in analyzing the bonding in extended systems from the viewpoint of molecular orbitals. ${ }^{60,61}$ The Wannier functions obtained from the 20 occupied valence states in bulk $\mathrm{SnS}$ are organized into three distinct types, shown in Figs. 3(a)-3(c), providing a useful picture of the type of chemical bonding present in the system: out of the 40 electrons in the unit cell, 8 of them are accommodated in a lone-pair type state that is present on Sn [Fig. 3(a)], 24 in a bonding type state between Sn-S pairs [Fig. 3(b)], and 8 in a state which is localized around S [Fig. 3(c)]. The Wannier analysis can yield further insight by examining how the
Wannier functions project onto the Bloch band structure. In Fig. 3(d), we illustrate the division of the Bloch bands into states which predominately project onto the Sn lone-pair and states which project onto the other two types of Wannier functions. The Sn lone-pair states contribute strongly to the states at the top of the valence band and states 5 to $8 \mathrm{eV}$ below the top of the valence band, whereas the other Bloch states are dominated by the bonding states and S-states.

To summarize, the electronic band structure and related electronic properties such as band gaps vary significantly with respect to the number of layers: as the number of layers is decreased, the splitting of the bands that delimit the band gap becomes less pronounced, the valence band maximum and conduction band minimum shifts lower and higher in energy, respectively, and the band gap widens. The reason for the gap increase is the reduced interaction between layers, which is mainly due to the interaction of $p$ orbitals across the interlayer region: less interaction of these orbitals leads to smaller band dispersion and larger band gap. This is mostly evident in the valence bands, which shows significantly less dispersion near the top in the monolayer than in bulk. As shown in Fig. 3, the Sn lone-pair states are directed outward into the interlayer region. These states should be quite sensitive to the presence of additional layers, the increased interaction between such states causing more dispersion and thus a smaller band gap as the number of layers is increased. A similar trend has been reported for transition metal dichalcogenides, ${ }^{25}$ although in that case an indirect-todirect band gap transition has also been identified, which is not observed in our models for $\mathrm{SnS}$. For $\mathrm{SnS}$, the indirect band gap is reduced by $1.50 \mathrm{eV}$ in going from the singlelayer to the bulk.

Another approach for tuning the properties of $\mathrm{SnS}$ is the application of strain. As a starting point, we study the elastic properties of the unstrained bulk structure. The bulk modulus and pressure derivative are $43 \mathrm{GPa}$ and 4.3 , respectively, using a stress-strain approach (tension/compression of the unit cell up to $0.5 \%$ ). The corresponding measured values are $36.6 \mathrm{GPa}$ and $5.5,{ }^{33}$ and the values of $35 \mathrm{GPa}$ and $41 \mathrm{GPa}$ have been reported for the bulk modulus in previous theoretical studies. ${ }^{12,28}$ However, these values do not readily 
describe the anisotropy in tension/compression of the crystal: the measured ${ }^{33}$ linear compressibilities parallel to the $a-, b$-, and $c$-axis are 12,36 , and 80 in units of $10^{-4} \mathrm{GPa}^{-1}$, respectively. To understand better how tensile and compressive strain along the $a$-axis affects the structural properties of $\mathrm{SnS}$, we compare two sets of structures where the $a$-axis has been elongated or compressed equally: in one set the $a$-coordinate of the atomic positions is scaled accordingly, and in the other set the relative positions of the atoms within each layer are fixed so only the interlayer spacing is varied. We define the strain as $\epsilon=\mathrm{a} / \mathrm{a}_{0}-1$, where a and $\mathrm{a}_{0}$ are the lattice constants of the $a$-axis for the strained and unstrained structures, respectively. By varying the strain in the range between -0.05 and 0.05 , we calculate the total energy of each structure in the second set of structures to be consistently lower than the energy of the corresponding equalvolume structure in the first set (the energy difference is $-0.17 \mathrm{eV}$ for $\epsilon=-0.05$ and $-0.05 \mathrm{eV}$ for $\epsilon=0.05$ ). These calculations, as well as the values of the linear compressibilities, suggest that strain affects the dimensions of the structure primarily via the interlayer spacing, ${ }^{33}$ so in the following we consider only variation in the separation of layers with fixed atomic positions in each layer and fixed in-plane lattice parameters. Although the assumption of incompressible layers is not appropriate for high strains, ${ }^{31,33}$ it allows us to make useful predictions for smaller strains.

Starting from the unstrained bulk $\mathrm{SnS}$ we compress the structure by decreasing the spacing between the layers up to $5 \%$ strain $(\epsilon=-0.05)$. Likewise, we stretch the material by increasing the interlayer distance until the strain reaches 5\% $(\epsilon=0.05)$. Fig. 4 summarizes the effect of the tension/compression on the direct [Fig. 4(a)] and indirect [Fig. 4(b)] band gaps. The indirect band gap varies in the narrow energy range between $0.88 \mathrm{eV}$ and $0.91 \mathrm{eV}$. Interestingly, the band gap becomes narrower under either tensile or compressive strain as two different pairs of $k$-points define the band gap: $\bar{Z}_{\mathrm{v}}(\mathrm{VBM})$ and $\overline{\mathrm{Y}}_{\mathrm{c}}(\mathrm{CBM})$ for compressive stress, and $\overline{\mathrm{Z}}_{\mathrm{v}}$ $(\mathrm{VBM})$ and $\Gamma(\mathrm{CBM})$ for tensile stress. The variation in energy is larger for the direct band gap, and is equal to $\sim 60 \mathrm{meV} / 1 \%$ strain. Similar to the shift observed between different indirect transitions, a shift is also found for the direct band gap at $\epsilon=0.05$ from $\bar{Y}$ to $\Gamma$ and the direct band gap starts to decrease [Fig. 4(a)]. We explain this transition as follows: as the strain changes from compressive into tensile the interlayer distance is increased and the overlap between the orbitals of atoms in neighboring layers is reduced, and vice versa. The effect is pronounced for orbitals with a significant component parallel to the strain direction as in the case of $\Gamma\left(83 \% p\right.$ Sn-levels of mostly $p_{\mathrm{a}}$ character, $9 \% s$ Sn-levels). The energy level at $\overline{\mathrm{Y}}_{\mathrm{c}}(69 \% p$ Sn-levels of mostly $p_{\mathrm{b}}$ character, 19\% $s$ S-levels) is affected less. Eventually, a shift takes places close to $\epsilon=0$ in the relative position in energy between the two $k$-points.

The trends in the band gaps of the double-layer SnS can be explained by similar arguments. The indirect band gap varies between $1.31 \mathrm{eV}$ and $1.65 \mathrm{eV}$. The direct band gap varies by $\sim 50 \mathrm{meV} / 1 \%$ strain. In general, the direct band gaps are more sensitive to strain than the indirect band gaps. ${ }^{34}$ Moreover, for both bulk and double-layer $\mathrm{SnS}$ the shift in valence band maximum is accompanied by a dramatic increase in the electron effective mass, with the electron becoming $\sim 5$ times heavier in structures under tensile strain [see also Figs. 2(d)-2(f) and Table I].

It is expected that the band gaps approach those of the single-layer structure as the layers are separated, although neither the indirect nor the direct band gap becomes as wide as that of the single-layer within the range of strains studied here, which suggests that the interaction between the layers and uniaxial strain is not linear. In conclusion, the electronic structure of SnS may be tuned not only by controlling on the number of layers but also using mechanical deformation.

We discuss next how the optical response of $\mathrm{SnS}$ depends on the number of layers and their separation. We calculate the absorption coefficient, $\alpha$, for light polarized in the direction of each structural axis as $\alpha(\omega)$ $=\varepsilon_{2}(\omega) \hbar \omega / n(\omega)$, where $n(\omega)$ is the refractive index, calculated from $n(\omega)=\left(\varepsilon_{1}(\omega)+\sqrt{\varepsilon_{1}(\omega)^{2}+\varepsilon_{2}(\omega)^{2}}\right) / 2$ with $\varepsilon_{1}(\omega)$ and $\varepsilon_{2}(\omega)$ the frequency-dependent real and imaginary parts of the dielectric function. The dielectric function was calculated within the framework of time-dependent density functional theory ${ }^{55}$ using the bootstrap approach of Sharma, Gross and co-workers. ${ }^{56}$ Fig. 5 shows the absorption coefficient for our model structures, averaged over all directions and for photon energies $\hbar \omega$ up to $4.0 \mathrm{eV}$. The absorption of each structure is significant $\left(\alpha>5 \times 10^{4} \mathrm{~cm}^{-1}\right)$ for photon energies higher than the corresponding direct band gap. For photon energies higher than $3 \mathrm{eV}$, absorption is almost constant for all structures with $\alpha \simeq 10^{6} \mathrm{~cm}^{-1}$. Since the material is highly anisotropic, we also investigate the dependence of
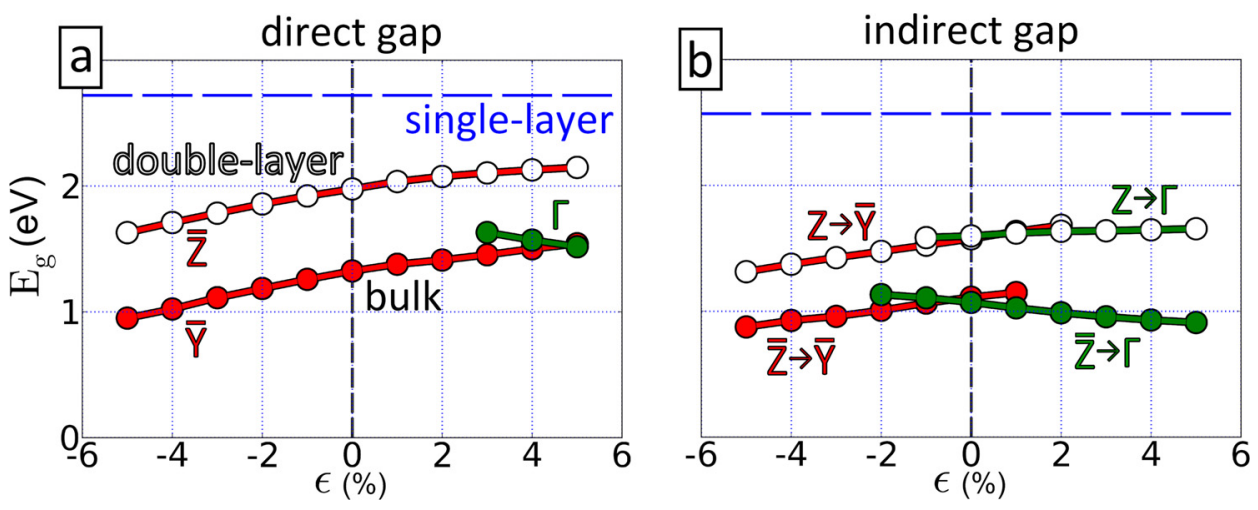

FIG. 4. Minimum band gap $\mathrm{E}_{\mathrm{g}}$ for (a) direct and (b) indirect transitions and associated band extrema in bulk (filled circles) and double-layer (empty circles) SnS with respect to strain, $\epsilon$, applied along the layer stacking direction. Negative and positive values of $\epsilon$ correspond to compressive and tensile strain, respectively, $(\epsilon=0$ for the unstrained $\mathrm{SnS}$ ). The horizontal dashed line marks the band gap of single-layer SnS. 

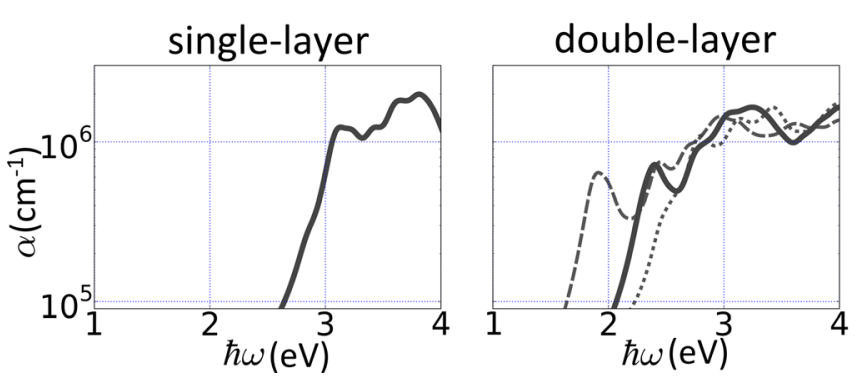

absorption on polarization. The anisotropy in the optical absorption of $\mathrm{SnS}$ is demonstrated in Fig. 6, which shows the imaginary part of the dielectric function for light polarized in the direction of each structural axis separately. The absorption edge for polarization along the $b$-axis lies lower in energy than for polarization along the $a$ - and $c$-axis, in accordance with the calculated direct band gaps. ${ }^{30}$ The maximum peak positions for polarization along the $a-, b-$, and $c$-axis are $\hbar \omega=2.5 \mathrm{eV}, 2.9 \mathrm{eV}$, and $3.9 \mathrm{eV}$, in agreement with measured positions at room temperature, that is, $2.8 \mathrm{eV}$ and $3.8 \mathrm{eV}$ for the $b$ - and $c$-axis. ${ }^{62}$ In comparison to the bulk structure, absorption is weaker for the double-layer $\mathrm{SnS}$. As for the bulk structure, the absorption edge for polarization along the $b$-axis lies at lower energy than for polarization along the $c$-axis and the position of the strongest peak for both the $b$ - and $c$-axis is at $3.0 \mathrm{eV}$, whereas two shorter peaks are found at $2.4 \mathrm{eV}$.

We also calculated static dielectric constants within the random phase approximation to illustrate better, by comparison, the effects of the electron-hole interaction as they are captured in the bootstrap approximation (Table II). In all cases, the corrected Kohn-Sham single-particle energies were used. Within the bootstrap approximation the mean value of the dielectric constants corresponding to the $a$-axis and $c$-axis is calculated to be 12.3 , in reasonable agreement with the experimental value of 12.5 at room temperature. ${ }^{10}$ In comparison, at the level of the random phase approximation, which neglects the electron-hole interaction, the dielectric constants are found to be systematically lower, for instance by 0.7 for the bulk SnS. A trend independent of the chosen approximation is found in the significant increase, by more than 2.5 times, of the dielectric constant in going from the single-layer to the bulk.

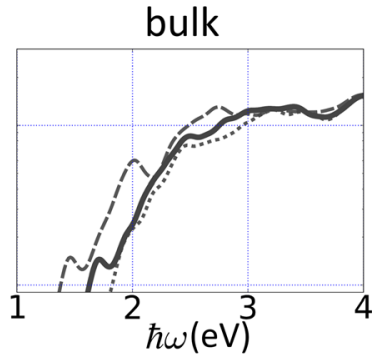

FIG. 5. Absorption coefficient, $\alpha$, as a function of photon energy, $\hbar \omega$, for the model single-layer, double-layer, and bulk structures of SnS. Solid, dashed, and dotted lines correspond to unstrained structures, and structures under 5\% compressive and 5\% tensile strain along the layer stacking direction (a-axis in Fig. 1).

Our earlier discussion on the relation between uniaxial strain and the band gap suggest that optical absorption also depends on the separation between the layers in bulk SnS. For example, as the band gap of bulk $\mathrm{SnS}$ narrows with increasing compressive strain (Fig. 4), enhanced optical absorption is expected. Indeed, we calculate stronger absorption for the compressed SnS (Fig. 5). For $\epsilon=-0.05, \varepsilon_{2}(\omega)$ for polarization along the $a$ - and $c$-axis shows two pronounced peaks below the absorption edge of the unstrained $\mathrm{SnS}$, and the strongest peaks in $\varepsilon_{2}(\omega)$ for polarization along the $b$-axis are shifted almost rigidly towards lower energies (Fig. 6). In general, the peaks become more pronounced with compressive strain. The double-layer $\mathrm{SnS}$ follows the same trends. On the other hand, tensile strain has a less pronounced effect. To summarize, in both bulk and double-layer $\mathrm{SnS}$, compressive strain in the range between $0 \%$ and $5 \%$ narrows the band gap and enhances the optical absorption.

\section{CONCLUSIONS}

We used first-principles total-energy calculations based on density functional theory to study electronic and optical properties, such as the indirect and direct band gaps, and the dielectric functions of structural models of $\mathrm{SnS}$, and how these properties depend on uniaxial strain. We verified and complemented previous findings related to bulk $\mathrm{SnS}$, and we explored the properties of model single-layer and doublelayer $\mathrm{SnS}$ : the calculated band gaps are wider for fewer layers $(2.72 \mathrm{eV}, 1.57 \mathrm{eV}$, and $1.07 \mathrm{eV}$ for single-layer, double-layer, and bulk $\mathrm{SnS}$, respectively) and increase with tensile strain along the layer stacking direction (by $\sim 55 \mathrm{meV} / 1 \%$ strain). We conclude from these results that either a restriction of the number of layers or the application of uniaxial
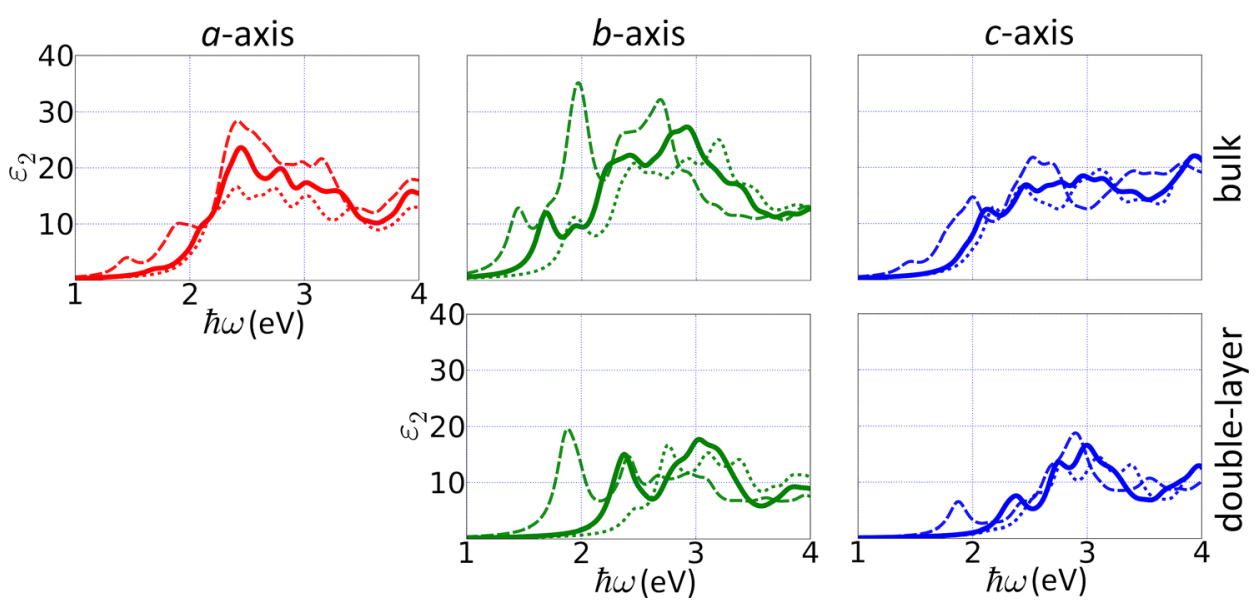

FIG. 6. Imaginary part of the dielectric function, $\varepsilon_{2}$, as a function of photon energy $\hbar \omega$ for the model bulk (top) and double-layer (bottom) structure of $\mathrm{SnS}$ with light polarized in the direction of each structural axis. Solid, dashed, and dotted lines correspond to unstrained structures, and structures under 5\% compressive and $5 \%$ tensile strain along the layer stacking direction $(a$ axis in Fig. 1). 
TABLE II. Static dielectric constants for the model single-layer, doublelayer, and bulk structures of $\mathrm{SnS}$, obtained within the random phase approximation and the bootstrap approximation.

\begin{tabular}{|c|c|c|c|c|c|c|}
\hline & \multicolumn{3}{|c|}{ Random phase approximation } & \multicolumn{3}{|c|}{ Bootstrap approximation } \\
\hline & $a$-axis & $b$-axis & $c$-axis & $a$-axis & $b$-axis & $c$-axis \\
\hline Single-layer & & 4.3 & 4.2 & & 4.9 & 4.8 \\
\hline Double-layer & & 6.9 & 6.3 & & 7.6 & 6.9 \\
\hline Bulk & 11.7 & 13.8 & 11.4 & 12.4 & 14.5 & 12.1 \\
\hline
\end{tabular}

strain could be used to obtain improvements in the performance of $\mathrm{SnS}$ as absorber material. Our findings suggest that the thickness of the $\mathrm{SnS}$ (one or two layers, or bulk) affects the properties of the material more drastically than applied strain. We suggest that both effects could be achieved by epitaxial growth on a substrate of a different lattice constant or by careful control of the amount of deposited material during atomic layer deposition.

\section{ACKNOWLEDGMENTS}

The authors wish to thank Prasert Sinsermsuksakul and Roy G. Gordon for the helpful discussions. Computations were performed on the Odyssey cluster, supported by the FAS Science Division Research Computing Group at Harvard University, the Extreme Science and Engineering Discovery Environment (XSEDE), which is supported by National Science Foundation grant number OCI-1053575, and the SEAS HPC cluster, supported by the Academic Computing Group.

${ }^{1}$ N. Armaroli and V. Balzani, Angew. Chem. Int. Ed. 46, 52 (2007).

${ }^{2}$ S. Chu and A. Majumdar, Nature 488, 294 (2012).

${ }^{3}$ P. Würfel, Physics of Solar Cells: From Basic Principles to Advanced Concepts (John Wiley \& Sons, 2009).

${ }^{4}$ M. A. Green, K. Emery, Y. Hishikawa, W. Warta, and E. D. Dunlop, Prog. Photovoltaics 20, 12 (2012).

${ }^{5}$ I. Repins, M. A. Contreras, B. Egaas, C. DeHart, J. Scharf, C. L. Perkins, B. To, and R. Noufi, Prog. Photovoltaics 16, 235 (2008).

${ }^{6}$ W. Albers, C. Haas, and F. van der Maesen, J. Phys. Chem. Solids 15, 306 (1960).

${ }^{7}$ A. P. Lambros, D. Geraleas, and N. A. Economou, J. Phys. Chem. Solids 35, 537 (1974).

${ }^{8}$ P. Sinsermsuksakul, J. Heo, W. Noh, A. S. Hock, and R. G. Gordon, Adv. Energy Mater. 1, 1116 (2011).

${ }^{9}$ M. M. El-Nahass, H. M. Zeyada, M. S. Aziz, and N. A. El-Ghamaz, Opt. Mater. 20, 159 (2002).

${ }^{10}$ A. M. Elkorashy, Phys. Status Solidi B 159, 903 (1990).

${ }^{11}$ N. K. Reddy and K. T. R. Reddy, Physica B 368, 25 (2005).

${ }^{12}$ J. Vidal, S. Lany, M. d' Avezac, A. Zunger, A. Zakutayev, J. Francis, and J. Tate, Appl. Phys. Lett. 100, 032104 (2012).

${ }^{13}$ B. Ghosh, M. Das, P. Banerjee, and S. Das, Sol. Energy Mater. Sol. Cells 92, 1099 (2008).

${ }^{14}$ C. Clemen, X. I. Saldaña, P. Munz, and E. Bucher, Phys. Status Solidi A 49, 437 (1978).

${ }^{15}$ H. Noguchi, A. Setiyadi, H. Tanamura, T. Nagatomo, and O. Omoto, Sol. Energy Mater. Sol. Cells 35, 325 (1994).

${ }^{16}$ K. T. Ramakrishna Reddy, N. Koteswara Reddy, and R. W. Miles, Sol. Energy Mater. Sol. Cells 90, 3041 (2006).

${ }^{17}$ P. D. Antunez, J. J. Buckley, and R. L. Brutchey, Nanoscale 3, 2399 (2011).

${ }^{18}$ J. B. Johnson, H. Jones, B. S. Latham, J. D. Parker, R. D. Engelken, and C. Barber, Semicond. Sci. Technol. 14, 501 (1999).
${ }^{19}$ I. Masaya, Sol. Energy Mater. Sol. Cells 93, 375 (2009).

${ }^{20} \mathrm{P}$. Sinsermsuksakul, R. Chakraborty, S. B. Kim, S. M. Heald, T. Buonassisi, and R. G. Gordon, Chem. Mater. 24, 4556 (2012).

${ }^{21}$ K. S. Novoselov, D. Jiang, F. Schedin, T. J. Booth, V. V. Khotkevich, S. V. Morozov, and A. K. Geim, Proc. Natl. Acad. Sci. U.S.A. 102, 10451 (2005).

${ }^{22}$ J. N. Coleman, M. Lotya, A. O’Neill, S. D. Bergin, P. J. King, U. Khan, K. Young, A. Gaucher, S. De, R. J. Smith, I. V. Shvets, S. K. Arora, G. Stanton, H.-Y. Kim, K. Lee, G. T. Kim, G. S. Duesberg, T. Hallam, J. J. Boland, J. J. Wang, J. F. Donegan, J. C. Grunlan, G. Moriarty, A. Shmeliov, R. J. Nicholls, J. M. Perkins, E. M. Grieveson, K. Theuwissen, D. W. McComb, P. D. Nellist, and V. Nicolosi, Science 331, 568 (2011).

${ }^{23}$ K. F. Mak, C. Lee, J. Hone, J. Shan, and T. F. Heinz, Phys. Rev. Lett. 105, 136805 (2010).

${ }^{24}$ C. Ataca, H. Şahin, and S. Ciraci, J. Phys. Chem. C 116, 8983 (2012).

${ }^{25}$ P. Johari and V. B. Shenoy, ACS Nano 6, 5449 (2012).

${ }^{26}$ B. Verberck, B. Partoens, F. M. Peeters, and B. Trauzettel, Phys. Rev. B 85, 125403 (2012).

${ }^{27}$ E. Scalise, M. Houssa, G. Pourtois, V. Afanas'ev, and A. Stesmans, Nano Res. 5, 43 (2012).

${ }^{28}$ H. C. Hsueh, H. Vass, S. J. Clark, G. J. Ackland, and J. Crain, Phys. Rev, B 51, 16750 (1995).

${ }^{29}$ L. Makinistian and E. A. Albanesi, Comput. Mater. Sci. 50, 2872 (2011).

${ }^{30}$ L. Makinistian and E. A. Albanesi, Phys. Status Solidi B 246, 183 (2009).

${ }^{31}$ G. Valiukonis, D. A. Guseinova, G. Keivaitb, and A. Sileika, Phys. Status Solidi B 135, 299 (1986).

${ }^{32}$ Z. Nabi, A. Kellou, S. Méçabih, A. Khalfi, and N. Benosman, Mater. Sci. Eng. 98, 104 (2003).

${ }^{33}$ L. Ehm, K. Knorr, P. Dera, A. Krimmel, P. Bouvier, and M. Mezouar, J. Phys.: Condens. Matter 16, 3545 (2004).

${ }^{34}$ M. Parenteau and C. Carlone, Phys. Rev. B 41, 5227 (1990).

${ }^{35}$ J. Y. Kim and S. M. George, J. Phys. Chem. C 114, 17597 (2010).

${ }^{36}$ M. Calixto-Rodriguez, H. Martinez, A. Sanchez-Juarez, J. CamposAlvarez, A. Tiburcio-Silver, and M. E. Calixto, Thin Solid Films 517, 2497 (2009).

${ }^{37}$ S. S. Hegde, A. G. Kunjomana, K. A. Chandrasekharan, K. Ramesh, and M. Prashantha, Physica B 406, 1143 (2011).

${ }^{38}$ B. Ghosh, M. Das, P. Banerjee, and S. Das, Appl. Surf. Sci. 254, 6436 (2008).

${ }^{39}$ M. Sugiyama, K. T. R. Reddy, N. Revathi, Y. Shimamoto, and Y. Murata, Thin Solid Films 519, 7429 (2011).

${ }^{40}$ W. S. Yun, S. W. Han, S. C. Hong, I. G. Kim, and J. D. Lee, Phys. Rev. B 85, 033305 (2012)

${ }^{41}$ T. Cao, G. Wang, W. Han, H. Ye, C. Zhu, J. Shi, Q. Niu, P. Tan, E. Wang, B. Liu, and J. Feng, Nat. Commun. 3, 887 (2012).

${ }^{42}$ W. Albers, C. Haas, H. J. Vink, and J. D. Wasscher, J. Appl. Phys. 32, 2220 (1961)

${ }^{43}$ J. Enkovaara, C. Rostgaard, J. J. Mortensen, J. Chen, M. Dułak, L. Ferrighi, J. Gavnholt, C. Glinsvad, V. Haikola, H. A. Hansen, H. H. Kristoffersen, M. Kuisma, A. H. Larsen, L. Lehtovaara, M. Ljungberg, O. Lopez-Acevedo, P. G. Moses, J. Ojanen, T. Olsen, V. Petzold, N. A. Romero, J. Stausholm-Møller, M. Strange, G. A. Tritsaris, M. Vanin, M. Walter, B. Hammer, H. Häkkinen, G. K. H. Madsen, R. M. Nieminen, J. K. Nørskov, M. Puska, T. T. Rantala, J. Schiøtz, K. S. Thygesen, and K. W. Jacobsen, J. Phys.: Condens. Matter 22, 253202 (2010).

${ }^{44}$ P. E. Blöchl, Phys. Rev. B 50, 17953 (1994).

${ }^{45}$ T. Olsen, J. Yan, J. J. Mortensen, and K. S. Thygesen, Phys. Rev. Lett. 107, 156401 (2011).

${ }^{46}$ O. Gritsenko, R. van Leeuwen, E. van Lenthe, and E. J. Baerends, Phys. Rev. A 51, 1944 (1995).

${ }^{47}$ M. Kuisma, J. Ojanen, J. Enkovaara, and T. T. Rantala, Phys. Rev. B 82, 115106 (2010).

${ }^{48}$ J. P. Perdew, A. Ruzsinszky, G. I. Csonka, O. A. Vydrov, G. E. Scuseria, L. A. Constantin, X. Zhou, and K. Burke, Phys. Rev. Lett. 100, 136406 (2008).

${ }^{49}$ J. Yan, K. W. Jacobsen, and K. S. Thygesen, Phys. Rev. B 86, 045208 (2012).

${ }^{50}$ I. E. Castelli, T. Olsen, S. Datta, D. D. Landis, S. Dahl, K. S. Thygesen, and K. W. Jacobsen, Energy Environ. Sci. 5, 5814 (2012).

${ }^{51}$ M. S. Hybertsen and S. G. Louie, Phys. Rev. B 34, 5390 (1986).

${ }^{52}$ W. G. Aulbur, L. Jönsson, and J. W. Wilkins, in Solid State Physics, edited by H. Ehrenreich and F. Spaepen (Academic Press, 1999), pp. 1-218.

${ }^{53}$ J. Deslippe, G. Samsonidze, D. A. Strubbe, M. Jain, M. L. Cohen, and S. G. Louie, Comput. Phys. Commun. 183, 1269 (2012). 
${ }^{54}$ P. Giannozzi, S. Baroni, N. Bonini, M. Calandra, R. Car, C. Cavazzoni, D. Ceresoli, G. L. Chiarotti, M. Cococcioni, I. Dabo, A. Dal Corso, S. de Gironcoli, S. Fabris, G. Fratesi, R. Gebauer, U. Gerstmann, C. Gougoussis, A. Kokalj, M. Lazzeri, L. Martin-Samos, N. Marzari, F. Mauri, R. Mazzarello, S. Paolini, A. Pasquarello, L. Paulatto, C. Sbraccia, S. Scandolo, G. Sclauzero, A. P. Seitsonen, A. Smogunov, P. Umari, and R. M. Wentzcovitch, J. Phys.: Condens. Matter 21, 395502 (2009).

${ }^{55}$ J. Yan, J. J. Mortensen, K. W. Jacobsen, and K. S. Thygesen, Phys. Rev. B 83, 245122 (2011)

${ }^{56}$ S. Sharma, J. K. Dewhurst, A. Sanna, and E. K. U. Gross, Phys. Rev. Lett. 107, 186401 (2011)
${ }^{57}$ S. Sharma, J. K. Dewhurst, A. Sanna, A. Rubio, and E. K. U. Gross, New J. Phys. 14, 053052 (2012).

${ }^{58}$ A. R. H. F. Ettema, R. A. de Groot, C. Haas, and T. S. Turner, Phys. Rev. B 46, 7363 (1992).

${ }^{59}$ F. M. Gashimzade, D. G. Guliev, D. A. Guseinova, and V. Y. Shteinshrayber, J. Phys.: Condens. Matter 4, 1081 (1992).

${ }^{60}$ N. Marzari, A. A. Mostofi, J. R. Yates, I. Souza, and D. Vanderbilt, Rev. Mod. Phys. 84, 1419 (2012).

${ }^{61}$ A. A. Mostofi, J. R. Yates, Y.-S. Lee, I. Souza, D. Vanderbilt, and N. Marzari, Comput. Phys. Commun. 178, 685 (2008).

${ }^{62}$ R. Eymard and A. Otto, Phys. Rev. B 16, 1616 (1977). 